\title{
Reliability and validity of the Mywellness Key physical activity monitor
}

This article was published in the following Dove Press journal:

Clinical Epidemiology

23 January 2013

Number of times this article has been viewed

\author{
John C Sieverdes' \\ Eric E Wickel ${ }^{2}$ \\ Gregory A Hand ${ }^{3}$ \\ Marco Bergamin ${ }^{4}$ \\ Robert R Moran 5 \\ Steven N Blair 3,5 \\ 'Medical University of South Carolina, \\ College of Nursing, Charleston, SC, \\ ${ }^{2}$ University of Tulsa, Exercise and \\ Sport Science, Tulsa, OK, ${ }^{3}$ University \\ of South Carolina, Department \\ of Exercise Science, Division of \\ Health Aspects of Physical Activity, \\ Arnold School of Public Health, \\ Columbia, SC, USA; ${ }^{4}$ University of \\ Padova, Department of Medicine, \\ Sports Medicine Division, Padova, \\ Italy; ${ }^{5}$ University of South Carolina, \\ Department of Epidemiology and \\ Biostatistics, Arnold School of Public \\ Health, Columbia, SC, USA
}

Background: This study evaluated the reliability and criterion validity of the Mywellness Key accelerometer (MWK) using treadmill protocols and indirect calorimetry.

Methods: Twenty-five participants completed two four-stage 20-minute treadmill protocols while wearing two MWK accelerometers. Reliability was assessed using raw counts. Validity was assessed by comparing the estimated $\mathrm{VO}_{2}$ calculated from the MWK with values from respiratory gas exchange.

Results: Good overall and point estimates of reliability were found for the MWK (all intraclass correlations $>0.93)$. Generalizability theory coefficients showed lower values for running speed $(0.70)$ versus walking speed (all $>0.84$ ), with the majority of the overall percentage of variability derived from the participant $(68 \%-88 \%$ of the total $100 \%)$. Acceptable validity was found overall (Pearson's $r=0.895-0.902, P<0.0001$ ), with an overall mean absolute error of $16.22 \%$ and a coefficient of variance of $16.92 \%$. Bland-Altman plots showed an overestimation of energy expenditure during the running speed, but total kilocalories were underestimated during the protocol by approximately $10 \%$.

Conclusion: Good validity was found during light and moderate walking, while running was slightly overestimated. The MWK may be useful for clinicians and researchers interested in promotion or assessment of physical activity.

Keywords: physical activity, accelerometer, health monitor

\section{Introduction}

Promotion of physical activity is an important aspect of public health in the prevention of morbidity and mortality in the United States and other nations. Accelerometry is a useful assessment tool for measuring physical activity in individual and populationbased studies, but only if the devices are valid and reliable. Even with the limitations for measuring whole body acceleration, ${ }^{1}$ accelerometers are a practical way of assessing physical activity objectively in interventions ${ }^{2,3}$ or in surveillance programs, ${ }^{4}$ such as the US National Health and Nutrition Examination Survey. ${ }^{5}$ Many validation studies have used various accelerometers in laboratory and field settings to assess activity levels in different populations. ${ }^{6-12}$

The Mywellness Key (MWK) has been introduced by Technogym SpA (Gambettola, Italy), and is a new consumer-based accelerometer. The device measures acceleration of movement using a uniaxial design and proprietary algorithms to determine the time and intensity when worn on clothing at a person's waist. Two studies have investigated the MWK, and have shown strong concurrent validity with the ActiGraph during treadmill $(\mathrm{r}=0.91, P<0.01)$ and free-living $(\mathrm{r}=0.73-0.76, P<0.01)$
Correspondence: John C Sieverdes Medical University of South Carolina, College of Nursing and Medicine, 99 Jonathan Lucas St, Room 424, Charleston, SC 29425, USA

Tel +l 8032405790

Email sieverde@musc.edu 
settings, ${ }^{13}$ while a second study measured criterion validity with gas exchange $(\mathrm{r}=0.94, P<0.001$; standard error of estimate $2.42 \mathrm{~mL} / \mathrm{kg}$ per minute), although reliability was not assessed. ${ }^{14}$ In addition, estimates and the variability of total energy expenditure against indirect calorimetry have not been evaluated for the MWK when assessing usability of the device. Therefore, this study evaluated the reliability and validity of the MWK during a range of exercise intensities to evaluate its utility as an objective physical activity monitor.

\section{Materials and methods}

Participants

Twenty-five participants were recruited from the University of South Carolina during 2011 using flyers posted at the campus. A convenience sample consisting of graduate students was used in this study. Inclusion criteria included both women and men with a body mass index of $18.5-29.9 \mathrm{~kg} / \mathrm{m}^{2}$, from any racial/ethnic group, who were physically able to walk briskly for 30 minutes and jog for at least 5 minutes. Height was limited to $148.6-185.4 \mathrm{~cm}$, corresponding to the 2.5th and 97.5 th percentile of height. ${ }^{15}$ We excluded people with gait abnormalities (limps or injuries that impeded walking or running); current heart disease, stroke, type 2 diabetes, and peripheral vascular disease; current pregnancy; and those taking medications that might affect circulation or heart rate response, eg, beta-blockers and hypertensive medication.

Height was measured to the nearest $\mathrm{cm}$ using a stadiometer (Ayrton Corporation, Model S100, Prior Lake, MN). Weight was measured using an electronic scale (Home Health Care Digital Scale, Model MC-660, C-7300 v1.1) to the nearest $0.1 \mathrm{~kg}$. Waist circumference was measured using a measurement tape $5 \mathrm{~cm}$ above the umbilicus to the nearest $0.5 \mathrm{~cm}$. Body fat percentage was assessed using the generalized gender-specific three-site skinfold test from the American College of Sports Medicine's guidelines for exercise testing and prescription. ${ }^{16}$

\section{Mywellness Key}

The MWK is a small, lightweight $\left(8.5 \times 2.0 \times 0.7 \mathrm{~cm}^{3}\right.$, $18.7 \mathrm{~g}$ ), consumer-grade uniaxial accelerometer designed to measure vertical accelerations of the human body when in motion. This device was designed to integrate data from recreation center exercise machines and free-living activity, when worn at the waist, into a single energy expenditure value. The sampling frequency of the device is $16 \mathrm{~Hz}$ and measures acceleration from 0.06 to $12.0 \mathrm{~g}$ ( $1 \mathrm{~g}=9.81$ meters per second ${ }^{2}$ ) with a frequency response from 0.1 to $5 \mathrm{~Hz}$.
Using an embedded algorithm, the MWK converts raw counts and outputs the physical activity level of an individual in real time during the course of the day to a liquid crystal display screen. The device uses a proprietary metric called "MOVE", which is unitless, to measure the total volume of physical activity. It is similar to a metabolic equivalent (MET) minute. The device also records and displays the time in light (1.8-2.9 METs), moderate (3-5.9 METs), or vigorous activity ( $\geq 6 \mathrm{METs}$ ). For this study, proprietary Technogym firmware and equations (firmware version 7190) were used to record counts directly from the device during the protocol in one-second epochs. This was converted into METs (conversion factor 3.5), $\mathrm{VO}_{2}$, and MOVE using Technogym algorithms.

\section{Testing protocol}

Each participant performed two identical treadmill tests. For the two tests, participants were randomly assigned two MWKs from a group of 10 supplied by the manufacturer. The first MWK was placed on a belt at the anterior of the right hip in line with the right knee. The second MWK was positioned directly above the first. The same MWKs were used at the same locations in both tests for each participant. The two tests were separated by at least 5 days.

The testing equipment consisted of a research-grade treadmill (Trackmaster, JAS, Model TMX42C, FullVision Inc, Newton, KS) and a Parvo gas analysis system (Parvo Medics, Sandy, UT). The treadmill protocol was computer-automated and consisted of a 2-minute warmup at $2.25 \mathrm{~km} /$ hour, and four 5 -minute stages at $3.22 \mathrm{~km} /$ hour, $5.96 \mathrm{~km} /$ hour, $6.44 \mathrm{~km} /$ hour, and $7.24 \mathrm{~km} /$ hour. The incline was set at $0 \%$ grade for all tests. Participants were required to walk during the first three stages, and jog during the last stage of the protocol. MET intensities were derived from American College of Sports Medicine treadmill speed formulae ${ }^{16}$ and corresponded to 2.52, 3.82, 4.76, and 7.90 METs for stages 1 through 4. An informed consent and medical screening was completed before starting the study protocol. The study was reviewed and approved by the University of South Carolina Institutional Review Board.

\section{Statistical analysis}

Descriptive statistics (mean \pm standard deviation) for age, height, weight, body mass index, waist circumference, and body fat percentages were calculated for all participants. Average counts and milliliters of oxygen consumption $\left(\mathrm{VO}_{2}\right)$ were used from the last 2 minutes of each stage for validity and reliability comparisons. 
Generalizability theory was used to partition and quantify sources of variance in MWK counts at each treadmill speed. ${ }^{17}$ In contrast with classical test theory, generalizability theory allows researchers to examine multiple sources of error and report reliability estimates systematically using a generalizability coefficient. ${ }^{18}$ In this study, a fully crossed design [participant $(\mathrm{P}) \times$ test $(\mathrm{T}) \times$ monitor $(\mathrm{M})$ ] was used to quantify the proportion of error. Intraclass correlation coefficients ${ }^{19}$ and coefficient of variance were used to assess the variation in reliability. In addition, repeated-measures analysis of variance were used for significance testing using Bonferroni adjustments.

Validity was assessed using mean absolute percent error and coefficient of variance between oxygen uptake measures and the MWK's estimated oxygen uptake computed from counts. Mean absolute percent error was used to describe absolute variation in the point estimates. Pearson's correlations were also used to test overall validity. ${ }^{20}$ Bland-Altman $95 \%$ limits of agreement ${ }^{21,22}$ were used to plot the bias of the results for individual and overall results, and to illustrate the spread of the data.

Comparisons of energy expenditure were tested using total computed kilocalories (kcals) from the 20-minute treadmill tests. The output from the Parvo for kcals was compared with a MWK-computed kcal value from the Technogym algorithm. Differences between the two devices were tested using a two-tailed Student's $t$-test with an alpha level of $P=0.05$. Accumulation of MET minutes from gas exchange and MOVE from the MWK were calculated from the average per minute values during the last 2 minutes of each stage, multiplied by the time in each stage ( 5 minutes), and combined. Statistical analyses were carried out using SAS version 9.2 (SAS Inc, Cary, NC) and SPSS version 19.0 (IBM, Armonk, NY).

\section{Results}

General demographics are reported in Table 1. The population was relatively young (27.6 \pm 4.5 years), and had low body fat $(15.2 \% \pm 7.8 \%)$. The study participants included men $(n=12)$ and women $(\mathrm{n}=13)$, who were normal to overweight (mean body mass index $22.4 \mathrm{~kg} / \mathrm{m}^{2}$, range $19.3-27.8 \mathrm{~kg} / \mathrm{m}^{2}$ ).

\section{Reliability}

Results from the generalizability theory analysis are shown in Table 2. The $\mathrm{T}$ term accounted for $<1 \%$ of the total variability, indicating consistency in mean MWK counts between testing trials at each speed. The relatively low proportion of error from the monitor term at each treadmill
Table I Descriptive characteristics of study participants

\begin{tabular}{lllll}
\hline Variables & Mean & SD & Minimum & Maximum \\
\hline Age (years) & 27.6 & 4.5 & 22 & 40 \\
Height $(\mathrm{cm})$ & 170.4 & 8.5 & 157.9 & 184.2 \\
Weight $(\mathrm{kg})$ & 65.3 & 10.5 & 49.6 & 92.6 \\
Waist circumference $(\mathrm{cm})$ & 72.8 & 6.3 & 63.8 & 87.3 \\
Body fat (\%) & 15.2 & 7.8 & 5.9 & 31.1 \\
BMI (kg/m $)$ & 22.4 & 2.5 & 19.3 & 27.8 \\
& $\mathbf{n}$ & $\%$ & & \\
Gender & & & & \\
$\quad$ Male & 12 & $48 \%$ & & \\
$\quad$ Female & 13 & $52 \%$ & & \\
$\quad$ Total & 25 & & & \\
Race & & & & \\
$\quad$ White & 20 & $80 \%$ & & \\
African American & 1 & $4 \%$ & & \\
Asian Indian & 2 & $8 \%$ & & \\
$\quad$ Other Asian & 2 & $8 \%$ & & \\
\hline
\end{tabular}

Notes: Body fat taken by three-site skinfold measurements. ${ }^{16}$ Waist circumference taken $5 \mathrm{~cm}$ above the navel.

Abbreviations: BMI, body mass index; SD, standard deviation.

speed suggests similar mean counts were obtained between monitor locations (top or bottom). The $\mathrm{T} \times \mathrm{M}$ interaction term contributed little toward the total variance, suggesting that the rank order of counts from each monitor location was similar between tests. The majority of the total variance in MWK counts was attributed to the mean differences between participants. The proportion of error from the participant term was relatively high across stages $1-3(84 \%-88 \%)$ and noticeably lower during stage 4 (68\%). Ideally, the participant term would account for the largest proportion of error, given that this is considered true error rather than "noise" from the instrumentation. The $\mathrm{T} \times \mathrm{P}$ interaction term represents the consistency in participant rank order between tests. Overall, slightly more variability in participant rank

Table 2 Generalizability theory reliability estimates for the Mywellness Key

\begin{tabular}{lllll}
\hline Variable & Stage I & Stage 2 & Stage 3 & Stage 4 \\
\hline Test $(\mathrm{T})$ & $0.00 \%$ & $0.00 \%$ & $0.00 \%$ & $0.67 \%$ \\
Monitor (M) & $0.00 \%$ & $0.44 \%$ & $0.74 \%$ & $2.44 \%$ \\
$\mathrm{~T} \times \mathrm{M}$ & $0.00 \%$ & $0.00 \%$ & $0.09 \%$ & $0.00 \%$ \\
Participants (P) & $84.15 \%$ & $86.65 \%$ & $88.32 \%$ & $67.75 \%$ \\
$\mathrm{~T} \times \mathrm{P}$ & $10.95 \%$ & $8.07 \%$ & $6.28 \%$ & $7.77 \%$ \\
$\mathrm{M} \times \mathrm{P}$ & $3.95 \%$ & $3.36 \%$ & $3.20 \%$ & $19.01 \%$ \\
$\mathrm{~T} \times \mathrm{M} \times \mathrm{P}$ & $0.95 \%$ & $1.49 \%$ & $1.37 \%$ & $2.35 \%$ \\
Total percentage & $100 \%$ & $100 \%$ & $100 \%$ & $100 \%$ \\
G reliability coefficient & 0.842 & 0.870 & $0.89 \mathrm{I}$ & 0.699 \\
SEM & 50.448 & 92.962 & $112.12 \mathrm{I}$ & 748.013 \\
\hline
\end{tabular}

Notes: Values represent \% of total source of variation for test $(T)$, monitor location $(M)$, participants $(P)$, and their interactions; $G$ reliability coefficient, computed from generalizability theory estimates of variation. Comparisons calculated from raw counts.

Abbreviation: SEM, standard error of the mean. 
order was observed at the slowest treadmill speed. The $\mathrm{M} \times \mathrm{P}$ interaction term describes the variability in participant rank order between monitor locations. The amount of error attributable to the $\mathrm{M} \times \mathrm{P}$ interaction was considerably higher in stage 4 compared with stages $1-3$ (19\% versus $3 \%-4 \%$, respectively). This finding emphasizes the need to standardize the monitor location during field and laboratory assessments. Overall, generalizability coefficients ranged from 0.84 to 0.89 for light and moderate walking, with a lower value found for the running stage (stage 4, 0.70).

Interunit reliability estimates are shown in Figure 1. The analyses resulted in acceptable values for Pearson's correlations for both tests $(r=0.97-0.98)$. When considering point estimates of reliability measures in each stage, correlations ranged from very good for stages $1-3$ (walking, $r=0.95-0.96)$ to borderline (running, $r=0.79-0.84$ ).
Intraclass correlation coefficients showed good values for reliability in each stage and when combined (minimum intraclass correlation coefficient of 0.93 ). The overall coefficient of variance was $11.87 \%$, with the highest amount of variability related to stage 4 (coefficient of variance 11.39 ), while other stages were lower $(2.81 \%-3.54 \%)$.

\section{Validity}

Validity was evaluated using Pearson's correlations, mean absolute percent error, coefficient of variance, and BlandAltman plots. Graphical representation and values for Pearson's correlations are displayed in Figure 2. The two tests resulted in a correlation of $\mathrm{r}=0.895-0.902$, which was greater than the criterion value of 0.85 . Total mean absolute percent error ( \pm standard deviation) for the MWK for all stages was $16.22 \% \pm 13.03 \%$ and the coefficient of

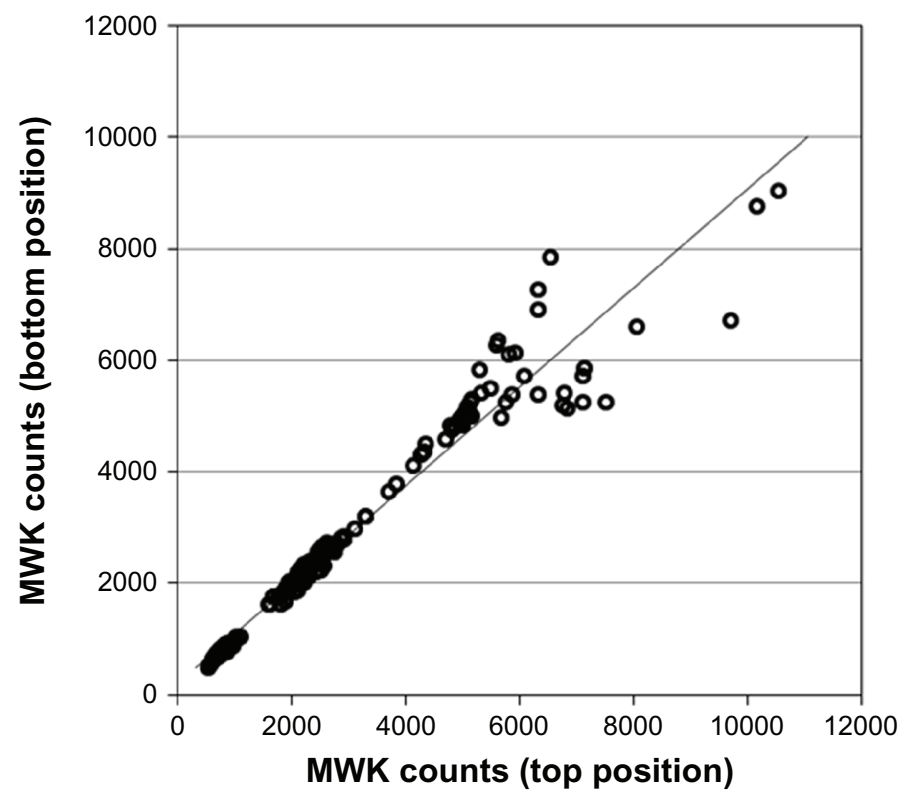

\begin{tabular}{|l|c|c|c|c|c|}
\hline & Stage 1 & Stage 2 & Stage 3 & Stage 4 & Stages 1-4 \\
\hline $\begin{array}{l}\text { Pearson's } \\
\text { coefficient, r }\end{array}$ & $\begin{array}{c}0.95-0.96 \\
P<0.0001\end{array}$ & $\begin{array}{c}0.95-0.96 \\
P<0.0001\end{array}$ & $\begin{array}{c}0.95 \\
P<0.0001\end{array}$ & $\begin{array}{c}0.79-0.84 \\
P<0.0001\end{array}$ & $\begin{array}{c}0.97-0.98 \\
P<0.0001\end{array}$ \\
\hline $\begin{array}{l}\text { ICC and 95\% } \\
\text { Cl }\end{array}$ & $\begin{array}{c}0.971 \\
(0.948-0.986)\end{array}$ & $\begin{array}{c}0.975 \\
(0.955-0.988)\end{array}$ & $\begin{array}{c}0.978 \\
(0.960-0.990)\end{array}$ & $\begin{array}{c}0.932 \\
(0.876-0.967)\end{array}$ & $\begin{array}{c}0.993 \\
(0.991-0.995)\end{array}$ \\
\hline CoV & $3.54 \%$ & $2.81 \%$ & $3.12 \%$ & $11.39 \%$ & $11.87 \%$ \\
\hline
\end{tabular}

Figure I Reliability coefficients for the MWK.

Notes: Data are represented as raw counts. The figure represents all tests and positions combined. Twenty-five participants completed two treadmill tests, each consisting of four stages. Pearson's coefficients ( $r$ ), 95\% confidence intervals, and $P$ values (Bonferroni adjusted) are reported between the top and bottom MWK positions for each test. Intraclass correlation coefficients were reported using data from the top and bottom placements for each test, for a total of four samples. Intraclass correlation coefficients were calculated using average measures and one-way random effects modeling with absolute agreement. The coefficient of variance was determined using all tests between top and bottom placements of the MWK.

Abbreviations: $\mathrm{Cl}$, confidence interval; ICC, intraclass correlation coefficient; MWK, Mywellness Key; CoV, coefficient of variance. 


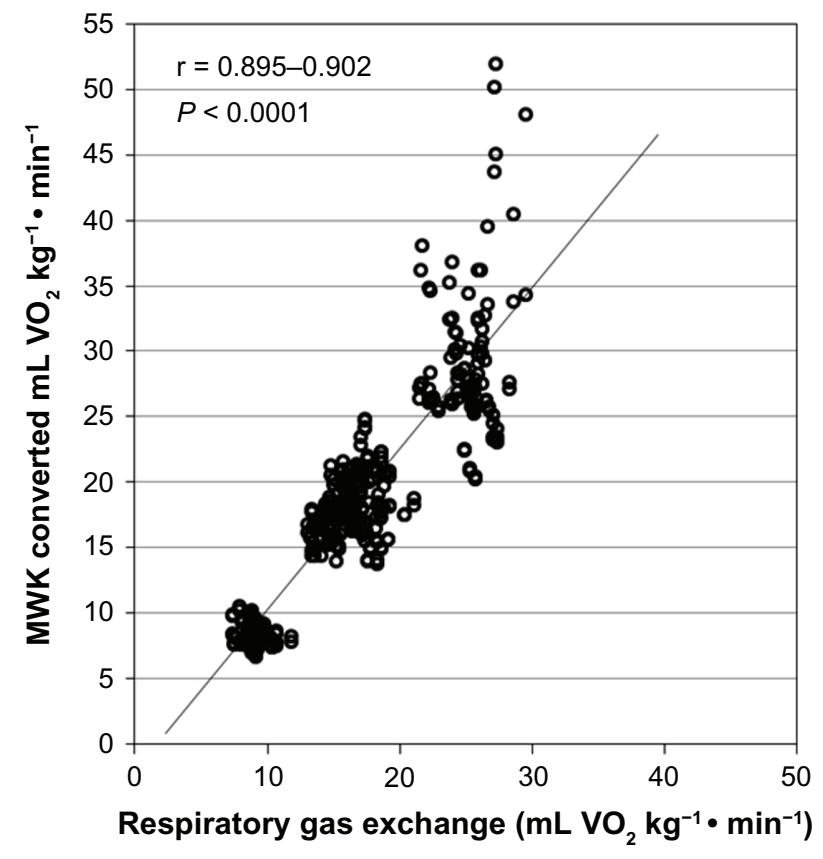

Figure 2 Validity of the MWK against gas exchange.

Notes: Pearson's correlations show $r$ values over two independent test times. $\mathrm{MWK}$ counts were converted to $\mathrm{VO}_{2}$ ( $\mathrm{mL} / \mathrm{kg}$ per minute). Each measurement poin represents the mean value during the last 2 minutes of the four-stage treadmill test. Abbreviation: MWK, Mywellness Key.

variance was $16.92 \%$ (Table 3), with higher values of mean absolute percent error found in the running stage (stage 4). Figure 3 shows Bland-Altman plots of all data. The overall mean and $95 \%$ limits of agreement was $1.8 \mathrm{~mL}(-5.6,9.3)$ $\mathrm{VO}_{2} / \mathrm{kg}$ per minute. The MWK was found to overestimate $\mathrm{VO}_{2}$ by $4.2 \mathrm{~mL}$ during stage 4 . The mean values for stages $1-3$ were close to 0 , ranging from $-0.5,1.5$, to $2.1 \mathrm{~mL}$, respectively. The limits of agreement were narrow for stage 1 and increased through stage 4 .

Figure 4 shows the comparisons between total energy expenditure (in kcals) derived from gas exchange and the MWK for the entire 20-minute exercise test protocol. Kcals between the two methods were significantly correlated

Table 3 Overall and point estimates of validity for the Mywellness Key

\begin{tabular}{llll}
\hline Stage & $\mathbf{n}$ & MAPE (SD) & CoV (\%) \\
\hline I light activity & 100 & $12.08 \% \pm 9.03 \%$ & 11.39 \\
2 moderate & 100 & $15.17 \% \pm 9.66 \%$ & 11.75 \\
3 moderate & 100 & $16.53 \% \pm 10.13 \%$ & 12.58 \\
4 vigorous & 100 & $21.10 \% \pm 19.08 \%$ & 18.59 \\
I-3 light + moderate & 300 & $14.60 \% \pm 9.77 \%$ & 12.60 \\
I-4 all stages & 400 & $16.22 \% \pm 13.03 \%$ & 16.92 \\
\hline
\end{tabular}

Notes: Assessments were calculated from estimated $\mathrm{VO}_{2}$ in $\mathrm{mL} / \mathrm{kg}$ per minute from the MWK compared with respiratory gas exchange values.

Abbreviations: $n$, sample size; MAPE, mean absolute percent error; MWK, Mywellness Key; SD, standard deviation; CoV, coefficient of variance.
( $\mathrm{r}=0.78, P<0.0001)$, with the MWK, underestimating energy expenditure by approximately $10.7 \mathrm{kcals}$ or $10.4 \%$, which was found to be significantly different $(P<0.001)$. Total MOVE was found to be correlated with gas exchange when each stage was used as separate data points $(\mathrm{r}=0.88$, $P<0.001)$. This implied a positive dose-effect accumulation of MOVE with intensity. Directly comparing MOVE with MET minutes showed an approximate ratio of 2.5 to 1 , with a good correlation between the variables $(r=0.90)$.

\section{Discussion}

In this analysis, we found high reliability (intraclass correlation coefficient 0.993 ) and good validity (Pearson's $\mathrm{r}=0.895-0.902$ ) for the MWK when measuring group physical activity, although the device showed moderate amounts of interindividual variability (mean absolute percent error $16.22 \%$, coefficient of variance $16.9 \%$ ). Point estimates for the MWK slightly overestimated gas exchange by $1.8 \mathrm{~mL}$ $\mathrm{VO}_{2}$ on average, with the estimated $\mathrm{VO}_{2}$ at the running stage being the most variable. MWK values for energy expenditure underestimated total energy expenditure by about $10 \%$. The error explained by generalizability theory was found to be derived mostly between participants and participant by monitor interactions, implying that variability between the devices is very low, and securing the MWK as critical in its application, especially at more vigorous speeds.

Overall, these results imply that the MWK could be useful in population-based physical activity studies for light and moderate activity, although individual results may show some variability, especially during vigorous activity. For reliability, we found that interindividual differences were more dependent on the placement of the device than other characteristics, such as weight or height, which did not contribute to excessive variability (data not shown). A suggested use for the MWK and other uniaxial accelerometers could be in physical activity programs that aim to increase minutes of light, moderate, and vigorous physical activity, with an emphasis on moderate activity. Using metrics from the real-time display may serve as a point of reference for individuals when trying to increase their minutes of a certain intensity or volume of activity as depicted by the MOVE metric. For estimating the amount of energy expenditure, users should be aware of the limitations of the MWK and other uniaxial accelerometers. Programming goals would be served by using the MOVE characteristic, which was found to correlate well with accumulation of physical activity across increasing levels of intensity. In our protocol, we found the ratio of MOVE to MET minutes 


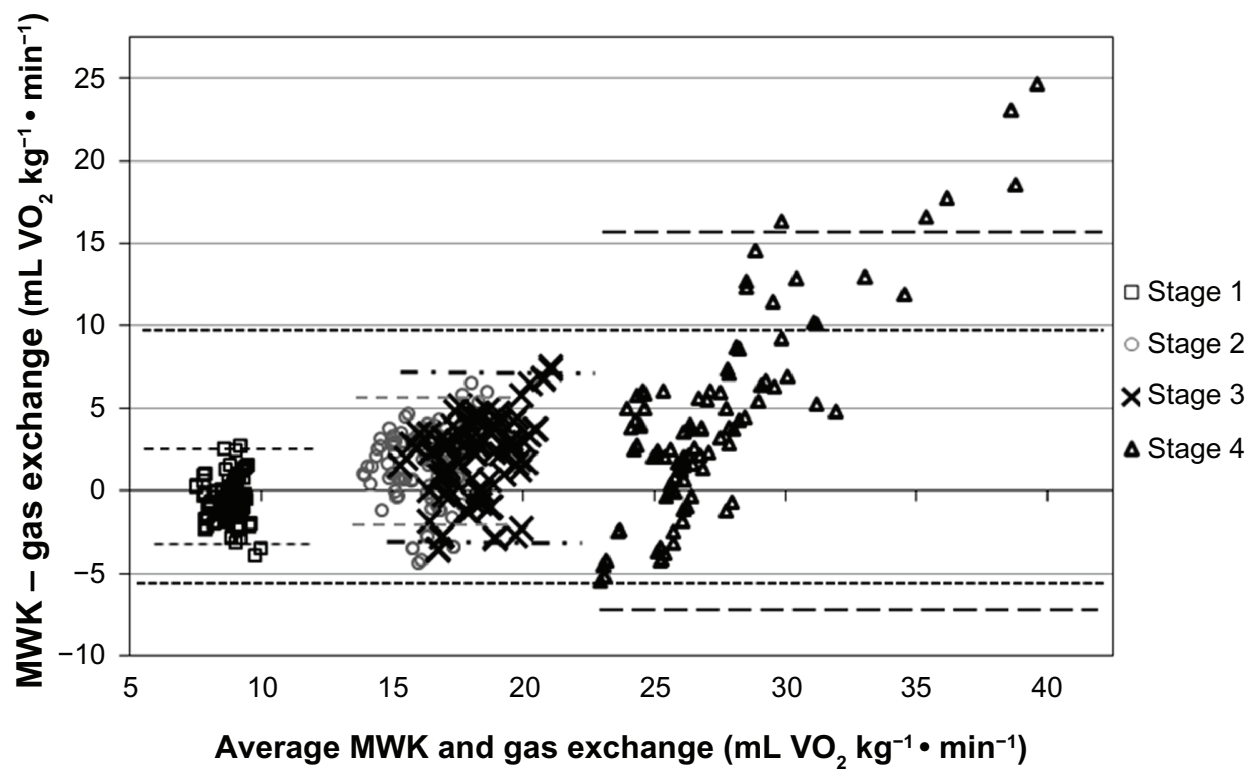

\begin{tabular}{|l|c|c|c|c|c|}
\hline & Stage 1 & Stage 2 & Stage 3 & Stage 4 & Stages 1-4 \\
\hline $\begin{array}{l}\text { Mean } \\
\text { difference } \\
\text { and 95\% } \\
\begin{array}{l}\text { limits of } \\
\text { agreement }\end{array}\end{array}$ & -0.5 & 1.5 & 2.1 & 4.2 & 1.8 \\
$(-3.1,2.0)$ & $(-2.8,5.9)$ & $(-2.7,6.9)$ & $(-7.3,15.7)$ & $(-5.6,9.3)$ \\
\hline
\end{tabular}

Figure 3 Bland-Altman plots of all data between converted MWK values and gas exchange $\left(\mathrm{VO}_{2}, \mathrm{~mL} / \mathrm{kg}\right.$ per minute).

Notes: Data values represent averages from the last 2 minutes of each stage for both MWK positions and tests ( $n=400)$, and $95 \%$ limits of agreement for overall and individual stages are represented.

Abbreviation: MWK, Mywellness Key.

to be about 2.5 to 1 . Five hundred to 1000 MET minutes, which is used as the range associated with substantial health benefits for adults, ${ }^{23,24}$ would equal approximately 1250-2500 MOVE units throughout a week.

Variability may be improved by incorporating calibration procedures for each specific user that take into account factors such as body weight, gait, and gender. Even though such analyses could not be performed in this study because of the proprietary nature of the device, tailoring the parameters of future or existing physical activity monitors to key personal characteristics may reduce interpersonal error and enhance the accuracy of output metrics for the end user.

\section{Comparison with other studies}

For more than two decades, many studies have assessed the utility of physical activity monitors, particularly accelerometers, but to the authors' knowledge, only two other studies have investigated the validity of the MWK. ${ }^{13}$ Hermann et al showed a strong association between the MWK and the ActiGraph for treadmill walking $(\mathrm{r}=0.91, P<0.01)$, but did not elaborate on variability at each testing stage. We found a similar but slightly lower correlation with gas exchange measures than that in the study reported by Bergamin et al $(\mathrm{r}=0.944, P<0.001)$ which was conducted in an Italian population. ${ }^{14}$ Similar measures were found between the MWK and other physical activity monitors. With the Caltrac accelerometer, the mean absolute percent error was found to be $23 \%$, which was greater than the values with the MWK (mean absolute percent error 16.2\%). ${ }^{10}$ They also found increasing levels of variation as treadmill speed increased, which we also observed for the MWK. The authors stated that the Caltrac overestimated energy expenditure, and the variation was possibly attributed to an interaction with each participant's fitness level. We found that the MWK also overestimated energy expenditure at similar intensities. In other studies, the ActiGraph and Kenz Lifecorder both significantly underestimated point estimates of energy expenditure at higher intensities when compared with indirect calorimetry $(P<0.001$ at $188 \mathrm{~m}$ per minute $),{ }^{6}$ although both devices were found to be accurate at slow to moderate walking speeds. Differences in the estimates could 


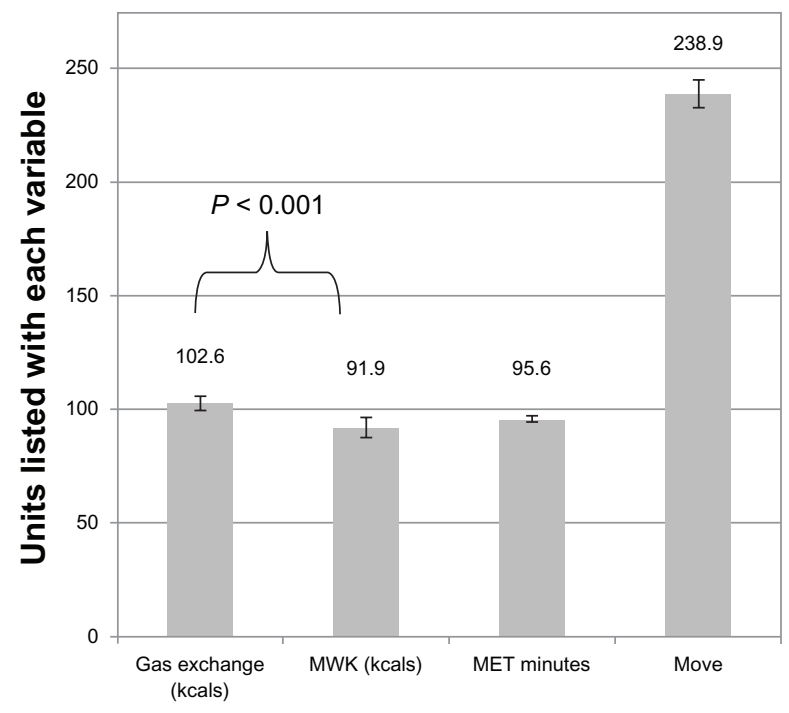

Figure 4 Total energy expenditure from gas exchange (kilocalories and calculated MET minutes), MWK, and MOVE characteristic over a 20-minute treadmill test protocol $(n=100)$.

Notes: The mean and $95 \%$ confidence interval of the mean are illustrated with bars. Comparisons between energy expenditure in kilocalories were tested using the Student's two-sided $t$-test with an alpha of $P=0.05$.

Abbreviations: MWK, Mywellness Key; MET, metabolic equivalents.

be attributed to the different algorithms used to express energy expenditure for the devices.

Reliability measures indicate that the MWK is similar to the ActiGraph, which has shown an intraclass correlation coefficient of 0.98 (95\% confidence interval $0.97-0.98))^{7}$ In the study with the ActiGraph, the device underestimated vigorous activity energy expenditure above $9 \mathrm{~km} / \mathrm{hour}$, which is in contrast with our study, in which the MWK overestimated energy expenditure. When compared with the Tritrac accelerometer, the MWK showed higher values for reliability (Tritrac, $r=0.87-0.92$ ), although validity, measured by indirect calorimetry, was slightly higher on the Tritrac $(r=0.94$, standard error of estimate $0.014 \mathrm{kcal} / \mathrm{kg}) .{ }^{12}$ The greatest source of variation for the reliability of the MWK was similar to findings in a study reported by Welk et al, where variation for the Actical, Biotrainer, Tritrac, and CSA/MTI ActiGraph was attributed to interindividual differences rather than interdevice differences. ${ }^{11}$ Low interdevice differences indicate that monitor to monitor variability is not normally a concern with research grade accelerometers, or as we found, with the MWK. Many of the differences between the devices may reflect differences in advancement of filtering or hardware between devices, the participants used, the test protocol, how the device was secured, or differences in the algorithms used. Design features for future models should consider three-dimensional outputs to minimize the error reflected by possible variability in positioning on the participant.

\section{Strengths and limitations}

In this study, the energy expenditure values only represent the average values for our participants and cannot easily account for interindividual differences in energy costs within the population with regard to mechanical efficiency or absolute work rates..$^{25}$ In fact, accelerometry data over a short time may not be representative of what a person usually does, ${ }^{26}$ and does not take into account walking on an incline, carrying loads, performing strength training, or exclusively upper body motion. Fortunately, human movement for healthy individuals is made up mostly of ambulatory activities, ${ }^{27}$ which is the principal activity measured by accelerometers. Our sample was limited to normal to overweight men and women. Assessment of individuals with higher body mass indexes or high body fat percentages may require additional validation due to increased variation in how the participant wears the device. The strengths of this study include the use of criterion gas exchange measures and generalizability theory to investigate multiple sources of variation, which have rarely been used in physical activity monitor studies. ${ }^{11,28}$ We suggest that validity and reliability studies should use generalizability theory to determine similar contributions to variation in other devices.

\section{Conclusion}

The MWK is an activity monitor that measures the amount of physical activity performed during free-living and structured exercise. This study examined the reliability and validity of the MWK during structured treadmill exercise and found it to be an adequate assessment device for population level measurements during slow or moderate locomotion speeds. For the MWK and other uniaxial accelerometers, minimizing the error for individuals' exercise intensity is challenging. Future designs should take into consideration calibrations to reduce interindividual error and triaxial designs to reduce error arising from placement of the monitor. The MWK may be useful in physical activity programs where increasing minutes in categories of physical activity or when the volume of physical activity is the main outcome. The MWK seems to be a good device for measuring population-based physical activity in normal to overweight younger adults, and has value-added potential by the incorporation of real-time feedback capability for use as an aid in health promotion.

\section{Acknowledgments}

We would like to thank Gregory Welk of Iowa State University, and Stewart Trost of Oregon State University 
for their valuable contribution to the methodological content of this paper. We would also like to thank Patrick Crowley sincerely for the data collection portion of this study, Sara Wilcox for her valuable input, and Silvano Zanuso for technical support.

\section{Disclosure}

This study was funded by Technogym, SpA, manufacturer of the Mywellness Key. The authors have no other conflicts of interest to declare.

\section{References}

1. Troiano RP, Freedson PS. Promises and pitfalls of emerging measures of physical activity and the environment. Am J Prev Med. 2010;38(6): 682-683.

2. Matthews CE, Wilcox S, Hanby CL, et al. Evaluation of a 12-week home-based walking intervention for breast cancer survivors. Support Care Cancer. 2007;15(2):203-211.

3. Pinto BM, Papandonatos GD, Goldstein MG, Marcus BH, Farrell N. Home-based physical activity intervention for colorectal cancer survivors. Psychooncology. September 9, 2011. [Epub ahead of print.]

4. Marquez DX, Hoyem R, Fogg L, Bustamante EE, Staffileno B, Wilbur J. Physical activity of urban community-dwelling older Latino adults. J Phys Act Health. 2011;8 Supp1 2:S161-S170.

5. Tudor-Locke C, Brashear MM, Johnson WD, Katzmarzyk PT. Accelerometer profiles of physical activity and inactivity in normal weight, overweight, and obese US men and women. Int J Behav Nutr Phys Act. 2010;7:60.

6. Abel MG, Hannon JC, Sell K, Lillie T, Conlin G, Anderson D. Validation of the Kenz Lifecorder EX and ActiGraph GT1M accelerometers for walking and running in adults. Appl Physiol Nutr Metab. 2008;33(6):1155-1164.

7. Brage S, Wedderkopp N, Franks PW, Andersen LB, Froberg K. Reexamination of validity and reliability of the CSA monitor in walking and running. Med Sci Sports Exerc. 2003;35(8):1447-1454.

8. Brooks AG, Gunn SM, Withers RT, Gore CJ, Plummer JL. Predicting walking METs and energy expenditure from speed or accelerometry. Med Sci Sports Exerc. 2005;37(7):1216-1223.

9. Nichols JF, Morgan CG, Chabot LE, Sallis JF, Calfas KJ. Assessment of physical activity with the Computer Science and Applications Inc, accelerometer: laboratory versus field validation. Res Q Exerc Sport. 2000;71(1):36-43.

10. Pambianco G, Wing RR, Robertson R. Accuracy and reliability of the Caltrac accelerometer for estimating energy expenditure. Med Sci Sports Exerc. 1990;22(6):858-862.
11. Welk GJ, Schaben JA, Morrow JR Jr. Reliability of accelerometrybased activity monitors: a generalizability study. Med Sci Sports Exerc. 2004;36(9):1637-1645.

12. Nichols JF, Morgan CG, Sarkin JA, Sallis JF, Calfas KJ. Validity, reliability, and calibration of the Tritrac accelerometer as a measure of physical activity. Med Sci Sports Exerc. 1999;31(6):908-912.

13. Herrmann SD, Hart TL, Lee CD, Ainsworth BE. Evaluation of the MyWellness Key accelerometer. Br J Sports Med. 2011;45(2): 109-113.

14. Bergamin M, Ermolao A, Sieverdes JC, Zaccaria M, Zanuso S. Validation of the MyWellness Key in walking and running speeds. J Sports Sci Med. 2012;11:57-63.

15. Tayyari F, Smith J. Occupational Ergonomics: Principles and Applications, 1st ed. New York, NY: Chapman \& Hall; 1997.

16. American College of Sports Medicine. ACSM's Guidelines for Exercise Testing and Prescription, Vol 8th ed. Philadelphia, PA: Lippincott Williams \& Wilkins; 2010.

17. Gleser GC, Cronbach LJ, Rajaratnam N. Generalizability of scores influenced by multiple sources of variance. Psychometrika. 1965;30(4): 395-418.

18. Brennan RL. Generalizability Theory. New York, NY: Springer; 2001.

19. Atkinson G, Nevill AM. Statistical methods for assessing measurement error (reliability) in variables relevant to sports medicine. Sports Med. 1998;26(4):217-238.

20. Dowda M, Pate RR, Sallis JF, et al. Agreement between student-reported and proxy-reported physical activity questionnaires. Pediatr Exerc Sci. 2007;19(3):310-318.

21. Chatburn RL. Evaluation of instrument error and method agreement. AANA J. 1996;64(3):261-268.

22. Bland JM, Altman DG. Statistical methods for assessing agreement between two methods of clinical measurement. Lancet. 1986;1(8476): 307-310.

23. US Department of Health and Human Services. 2008 Physical Activity Guidelines for Americans. 2008. Available from: http://www.health. gov/paguidelines/guidelines/default.aspx. Accessed October 1, 2012.

24. US Department of Health and Human Services. Department of Health and Human Services. Physical Activity Guidelines Advisory Committee Report. 2008. Available from: http://www.health.gov/PAGuidelines/ Report/. Accessed October 1, 2012.

25. Ainsworth BE, Haskell WL, Leon AS, et al. Compendium of physical activities: classification of energy costs of human physical activities. Med Sci Sports Exerc. 1993;25(1):71-80.

26. Gretebeck RJ, Montoye HJ. Variability of some objective measures of physical activity. Med Sci Sports Exerc. 1992;24(10):1167-1172.

27. US Bureau of Labor Statistics. BLS Spotlight on Statistics: Sports and Exercise. May 2008. Available from: http://www.bls.gov/spotlight/2008/ sports/pdf/sports_bls_spotlight.pdf. Accessed February 15, 2012.

28. Wickel EE, Welk GJ. Applying generalizability theory to estimate habitual activity levels. Med Sci Sports Exerc. 2010;42(8):1528-1534.
Clinical Epidemiology

\section{Publish your work in this journal}

Clinical Epidemiology is an international, peer-reviewed, open access journal focusing on disease and drug epidemiology, identification of risk factors and screening procedures to develop optimal preventative initiatives and programs. Specific topics include: diagnosis, prognosis, treatment, screening, prevention, risk factor modification, systematic Submit your manuscript here: http://www.dovepress.com/clinical-epidemiology-journal

\section{Dovepress}

reviews, risk \& safety of medical interventions, epidemiology \& biostatical methods, evaluation of guidelines, translational medicine, health policies \& economic evaluations. The manuscript management system is completely online and includes a very quick and fair peer-review system, which is all easy to use. 